\title{
The data universe of structural biology
}

\author{
Helen Berman ${ }^{1,2}$ \\ ${ }^{1}$ Rutgers University, United States of America; ${ }^{2}$ University of Southern California, United States of America; \\ berman@rcsb.rutgers.edu
}

The Protein Data Bank (PDB) has grown from a small data resource for crystallographers to a worldwide resource a very broad community of researchers and educators. In this talk I will describe the history of the growth of the PDB and the role that the community has played in developing standards and policies. I also present examples of how other biophysics communities are collaborating with the worldwide PDB to create a network of interoperating data resources. This network will expand the capabilities of structural biology and enable the determination of increasingly complex structures.

Keywords: structural biology, data resources 